\title{
Acute Kidney Injury Complicating the Management of Fatal Cerebral Edema after Cannabinoid Use - Case Report and Literature Review
}

Varun Shah*, Alok Dabi*

Department of Neurology, University of Texas Medical Branch, Galveston Texas

*Corresponding Author: Varun Shah, Department of Neurology, University of Texas Medical Branch, Galveston Texas Received date: June 04 2020; Accepted date: June 29, 2020; Published date: July 02, 2020

Citation: Varun S, Alok D. (2020) Acute Kidney Injury Complicating the Management of Fatal Cerebral Edema after Cannabinoid Use - Case Report and Literature Review. International Journal of Clinical Case Reports and Reviews. 2(5); DOI: 10.31579/2690-4861/028

Copyright: (c) 2020 Varun Shah, This is an open-access article distributed under the terms of the Creative Commons Attribution License, which permits unrestricted use, distribution, and reproduction in any medium, provided the original author and source are credited.

\section{Abstract}

Case Description: 19-year-old man with history of marijuana and Synthetic Cannabinoids use presented with status epilepticus. Labs showed tetrahydrocannabinol in urine. Imaging showed diffuse cerebral edema with non-traumatic subarachnoid hemorrhage. Intracranial pressure (ICP) was elevated to $30 \mathrm{mmHg}$. This was complicated by severe refractory metabolic acidosis with persistently elevated ICP unresponsive to medical care and cerebrospinal fluid drainage. Five days later patient suffered cardiac arrest from severe acidosis and metabolic derangements. Patient passed away after failed attempts to resuscitate.

Discussion: This case highlights the many challenges in managing a critically ill patient with cerebral edema and renal failure with medically refractory metabolic acidosis. SCs are undetectable on routine drug screens and exposure is difficult to establish. SCs can lead to multi-organ failure and death that may result from cardiovascular events, respiratory depression, pulmonary complications and AKI. A high clinical suspicion is warranted in at-risk patients.

Conclusion: Clinicians should always consider SCs use in high-risk patient and anticipate multi-organ damage for early intervention. Further research is needed to establish causality, improve detection and devise appropriate therapeutic strategies in managing such patients.

Keywords: neurocritical care; cannabis; cannabinoid; acute kidney injury; cerebral edema

\section{Introduction}

The prevalence of cannabis (marijuana) and synthetic cannabinoids (SCs) is increasing in recent times due to an international push towards its legalization. Admissions to the Neurocritical Care units from cannabis and/or cannabinoid related adverse health events are increasing. While acute neurological symptoms are common and well-documented in the literature[1], it's important that Neurologists familiarize themselves with the entire spectrum of ill-effects of the cannabinoids as involvement of multiple organ systems can complicate the management of a primary neurological problem in a critically ill patient. Particularly, acute kidney injury is under-reported with synthetic cannabinoid use and can complicate the management of a critically ill neurology patient.

\section{Case Description}

A 19-year-old Caucasian male with known cannabis and SCs abuse was admitted to our institution after new-onset seizures. History was positive for headaches, sleep deprivation and recent marijuana use. He was intubated emergently followed by intravenous levetiracetam and naloxone administration. However, clinical exam remained unchanged and was noted to have restlessness, inability to follow commands and preserved brainstem reflexes. Urine drug screen was positive for delta-9tetrahydrocannabinol (THC). Non-contrast CT Head showed diffuse cerebral edema with cortical sulcal hyperdensities (Figure 1) suspicious for subarachnoid blood and CT angiogram of head and neck was unremarkable for aneurysm or other vascular malformations. 


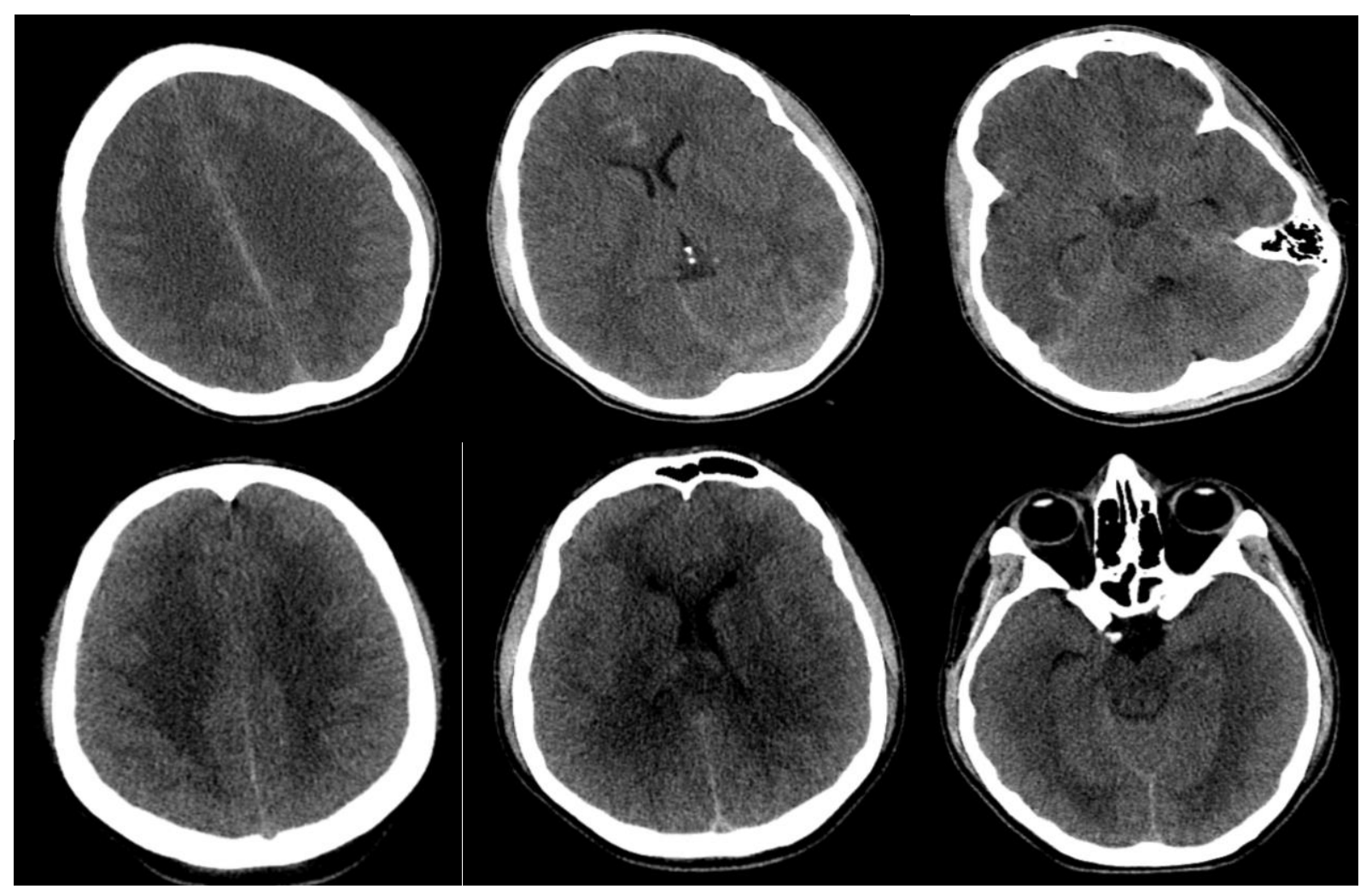

Figure 1 (A-F): Axial non-contrast CT Head. A-C represent admission images. D-F represent images at day 5 of admission. A) There is gyral fullness secondary to generalized cerebral edema. B) Hyperdensiites along the anterior inter-hemispheric fissure is suggestive of subarachnoid blood. C) Absence of normal gyrification with fullness of intracranial contents representing diffuse swelling. D) Persistent diffuse cerebral edema despite best medical care. E) Anterior inter-hemispheric hyperdensities have resolved. F) Obliteration of the ambient cisterns around the midbrain suggests impending uncal herniation.

Neurosurgery was consulted and an external ventricular drain (EVD) placement revealed elevated intracranial pressure (ICP) around 30 mmHg. Hyperosmolar therapy was immediately initiated to maintain serum Sodium between 145 and $155 \mathrm{mEq} / \mathrm{L}$. There was initial radiographic improvement in cerebral edema without clinical change. Patient developed acute kidney injury (AKI) with a steady deterioration in glomerular filtration rate, metabolic acidosis, hypocalcemia, and hyperkalemia. AKI made ICP management difficult as hyperosmolar therapy could not be titrated up given risk for electrolyte derangement and volume overload. Transient neuromuscular blockade was tried with transient drop in ICP. EVD was exchanged but showed consistent results with ICP more than $30 \mathrm{mmHg}$. Nephrology was consulted for continuous renal replacement therapy to stabilize him for a surgical decompression. However, the patient developed ventricular fibrillation. Return of spontaneous circulation was achieved after 15 minutes of resuscitation but then shortly after he had a second cardiac arrest with asystole. Family decided to abort resuscitative measures and he was declared dead subsequently.

\section{Discussion}

Out of the five-hundred compounds extracted from Cannabis, sixty-five are classified as cannabinoids. SCs abuse can be difficult to establish unless self-reported, as they are not detectable by standard lab tests. A high clinical suspicion is warranted in such at-risk patients. Their abuse can lead to multi-organ failure and even death that may result from cardiovascular events, respiratory depression, pulmonary complications or AKI.
There is a report of cerebral edema in three fatal cases from Germany with post-mortem drug analysis showing SCs from various body tissues [2]. However, there are no case reports of SCs related combination of cerebral edema, refractory renal failure with metabolic acidosis and new onset seizure as found in the case being reported by our team.

A rare but clinically significant adverse effect of Cannabis consumption that is under-reported in the literature includes acute kidney injury. Both CB1 and CB2 receptors are expressed in kidneys. In animal models of $\mathrm{AKI}, \mathrm{CB}$ receptor activation is associated with increased production of reactive oxygen species, which can either activate NF- $\kappa \beta$-dependent transcription of downstream proinflammatory target genes, or alternatively activate p38 MAPK and JNK. In the end, both pathways activate programmed cell death by apoptosis [3].

As demonstrated above, in addition to direct nephrotoxicity, the severe refractory metabolic acidosis from AKI associated with synthetic cannabis abuse can prove to be fatal. This case highlights the many challenges in managing a critically ill patient with cerebral edema and renal failure with medically refractory metabolic acidosis. Having a low threshold for suspecting cerebral edema and renal failure may potentially help save other patients who may have a similar clinical presentation. Unfortunately, as the use and abuse of the Marijuana gets more socially acceptable, similar presentations are likely to become more common.

\section{Conclusion:}

The spectrum of physiological and pathological effects of Cannabis and synthetic cannabinoids is not yet fully understood. Due to the increasing acceptance of marijuana in society-at-large, use of Cannabis is likely to become more prevalent. The perceived benefits of medical marijuana 
need to be compared with the known and potential health risks, both short and long-term, before accepting it at a community level [4]. Synthetic marijuana detection and control of natural marijuana contamination by appropriate use of legal and judicial measures needs to be strictly placed to avoid serious health consequences, especially to the younger generation. Further large-scale studies are warranted to evaluate the impact of this upcoming health challenge to the physicians and the society.

\section{References:}

1. K. Cohen and A. M. Weinstein. (2018) "Synthetic and Nonsynthetic Cannabinoid Drugs and Their Adverse Effects-A Review From Public Health Prospective," (in eng), Frontiers in public health, vol. 6, pp. 162-162, doi: 10.3389/fpubh.2018.00162.
2. V. Angerer, S. Jacobi, F. Franz, V. Auwärter, and J. Pietsch. (2017) "Three fatalities associated with the synthetic cannabinoids 5F-ADB, 5F-PB-22, and AB-CHMINACA," (in eng), Forensic science international, vol. 281, pp. e9-e15, doi: 10.1016/j.forsciint.2017.10.042.

3. P. Mukhopadhyay et al. (2010) "CB1 cannabinoid receptors promote oxidative/nitrosative stress, inflammation and cell death in a murine nephropathy model," (in eng), British journal of pharmacology, vol. 160, no. 3, pp. 657-668, doi: 10.1111/j.1476-5381.2010.00769.x.

4. J. L. Rein and C. M. Wyatt. (2018) "Marijuana and Cannabinoids in ESRD and Earlier Stages of CKD," (in eng), American journal of kidney diseases : the official journal of the National Kidney Foundation, vol. 71, no. 2, pp. 267-274, doi: 10.1053/j.ajkd.2017.06.020.
Ready to submit your research? Choose Auctores and benefit from:

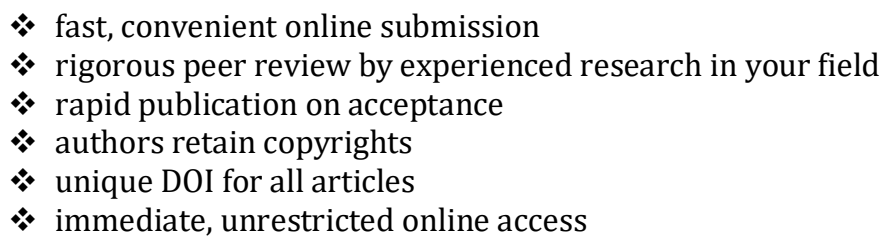

At Auctores, research is always in progress.

Learn more https://www.auctoresonline.org/journals/internationaljournal-of-clinical-case-reports-and-reviews 